\title{
Análisis de la sequía meteorológica en cuatro localidades agrícolas de Venezuela mediante la combinación de métodos multivariados
}

\author{
Barlin Orlando Olivares ${ }^{1} \&$ M. L. Zingaretti ${ }^{2}$ \\ 1. Investigador. Programa Iberoamericano de Doctores en Agroalimentación, Universidad de Córdoba (UCO), España; barlinolivares@gmail.com \\ 2. Docente- investigador. IAPCBA-IAPCH, Universidad Nacional de Villa María, Córdoba, Argentina.
}

Recibido 14-VIII-2017 • Corregido 19-X-2017 • Aceptado 20-XI-2017

\begin{abstract}
Analysis of the meteorological drought in four agricultural locations of Venezuela by the combination of multivariate methods. On a global scale, agriculture is considered the most vulnerable to drought, representing one of the most devastating climate threats. For this reason, the objective of this work was to analyze the occurrence of meteorological drought by means of time series of the Normalized Precipitation Index during the period (1980-2014) for four Venezuelan agricultural sites. For the analysis, we used the combination of two multivariate methods: the Principal Coordinate Analysis of the data matrices using Euclidean Distance and the Cluster Analysis. The results of the analysis revealed three groups of years for the localities El Tigre and Banco de San Pedro, represented by the wet years, normal or intermediate conditions and years with significant water deficit; while for the rest of the localities (Tapipa-Padrón and El Guayabo) only two groups of years are distinguished (humid and dry). The statistical methodology based on the multivariate analysis allowed delineating groups of years that showed similar patterns of the index of meteorological drought as a function of the time series. The study is the backbone for space planning and the strengthening of strategies for food security in those localities.
\end{abstract}

Key words: agriculture, climate, precipitation, water deficit, drought
RESUMEN: A escala global, la agricultura es considerada la actividad más vulnerable a la sequía, representando una de las amenazas climáticas más devastadoras. Es por ello que, el objetivo de este trabajo fue analizar la ocurrencia de la sequía meteorológica por medio de series temporales del Índice Normalizado de Precipitación durante el periodo (1980-2014) para cuatro localidades agrícolas venezolanas. Para el análisis, se usó la combinación de dos métodos multivariados: el Análisis de Coordenadas Principales de las matrices de datos usando distancia Euclídea y el Análisis Clúster. Los resultados del análisis revelaron tres grupos de años para las localidades El Tigre y Banco de San Pedro, representados por los años húmedos, condición normal o intermedios y años con déficit hídrico significativo; mientras que para el resto de las localidades (Tapipa-Padrón y El Guayabo) solo se distinguen dos grupos de años (húmedos y secos). La metodología estadística basada en el análisis multivariado permitió delinear grupos de años que mostraron patrones semejantes del índice de sequía meteorológica en función a la serie temporal. El estudio constituye la columna vertebral para la planificación del espacio y el fortalecimiento de las estrategias para la seguridad alimentaria en esas localidades.

Palabras clave: agricultura, clima, precipitación, déficit hídrico, sequía
La sequía es considerada como una manifestación dramática de la variabilidad del ciclo hidrológico del planeta y uno de los fenómenos climáticos más complejos que afectan a la sociedad y el medio ambiente (Wilhite, 2000; Bonsal, Wheaton, Chipanshi, Lin, Sauchyn \& Wen, 2011). Las sequías están afectando a una mayor superficie de Tierra, especialmente en las zonas tropicales y en latitudes medias. En las próximas décadas, se espera que las sequías sean frecuentes y severas por el cambio climático (Ovalles, Cortez, Rodríguez, Rey \& Cabrera-Bisbal, 2008).

En cierto modo, la sequía difiere en distintos aspectos de otros fenómenos naturales perjudiciales tales como ciclones, inundaciones y tormentas; sus efectos se hacen notar de manera muy pausada o lenta. Es decir, la sequía es una desviación acumulativa respecto de las condiciones normales de precipitación (Wilhite \& Glantz, 1985).

Este déficit de precipitación puede sobrevenir en poco tiempo o tardar meses en manifestarse por medio de la disminución del caudal de los ríos, de los niveles de los embalses o del nivel freático, y esa evolución sigilosa de la sequía hace que, sus efectos tarden semanas o meses en hacerse presentes (Wilhite, 1997).

Los cultivos de secano, concentrados en las regiones tropicales y subtropicales, generan casi el $40 \%$ de los 
alimentos básicos en el mundo a más de 2 mil millones de personas, siendo esta producción agrícola sumamente afectada por la ocurrencia de eventos extremos como las sequías (FAO, 2003).

En el caso particular de Venezuela, el territorio nacional ha atravesado a lo largo de la historia por eventos de intensa sequía, producto del inicio tardío del período lluvioso, tal como lo reportan los estudios desarrollados por Hernández (2008); Paredes, Millano y Guevara (2008); Guevara y Paredes (2007); Paredes y Guevara (2010); Olivares, Cortez, Lobo, Parra, Rey y Rodríguez (2016a); Quiroz, Paredes y Guevara (2016). Esto generó el racionamiento de agua en varias regiones, para cuidar las reservas de los embalses más importantes, pero también afectó en mayor magnitud al sector agropecuario y la producción de alimentos en el país.

En el país, a excepción del sur deVenezuela (Amazonas), el régimen climático presenta dos períodos, uno seco que va de noviembre hasta marzo y otro lluvioso desde abril hasta inicio de octubre, siendo los meses de abril y octubre conocidos como de transición (Cárdenas, García \& Gil, 2002; Martelo, 2003, Rojas \& Alfaro, 2000).

Es evidente que la crisis de agua que existe en Venezuela no solamente está afectando la vida cotidiana de sus habitantes, sino que también está incidiendo negativamente en las actividades agrícolas y pecuarias de zonas que son consideradas importantes en la producción y seguridad alimentaria de la nación, lo cual puede agravar la ya difícil situación con la escasez de alimentos. Paralelamente, la sequía es un conductor de la desertificación, o la degradación del suelo en las tierras secas (Olivares, 2017).

Por otra parte, se plantea entonces el problema asociado a que en el país existe información en un nivel muy general sobre la sequía, pero aún no se han concentrado esfuerzos por constituir estudios detallados sobre este fenómeno meteorológico y las medidas de adaptación o prevención, sobre todo en entornos rurales y agrícolas, los cuales podrían servir de base para comprender en un sentido más amplio el problema de la desertificación y sus relaciones con la sequía (Paredes \& Guevara, 2010).

El Índice Normalizado de Precipitación (SPI, siglas en inglés) fue desarrollado por Mckee, Doesken y Kleist (1993) con la finalidad de mejorar la detección del inicio y el monitoreo de la evolución de las sequías meteorológicas (definidas únicamente en función de la precipitación). Se escogió este índice por la simplicidad del cálculo, dado que está basado solamente en la precipitación. También, puede ser calculado para escalas de tiempo variables, lo cual lo hace aplicable en el ámbito de la meteorología, agricultura e hidrología superficial y subterránea. Esta versatilidad temporal es particularmente útil en el análisis de la dinámica de la sequía (al igual que la de los períodos con excesos hídricos), especialmente en la determinación del comienzo y el fin de estos episodios, lo que es difícil de reconocer por medio de otros índices.

De modo general, su cálculo se resume en dos etapas: la primera consiste en ajustar la serie histórica de la precipitación a una distribución teórica Gamma. La segunda etapa consiste en convertir la serie de precipitación ajustada a la distribución Gamma en una distribución normal estandarizada con media (0) y varianza (1), cuyo resultado representa el índice SPI (Edwards \& McKee, 1997). Dado que los registros nulos en las subseries mensuales imposibilitan calcular la variable auxiliar adimensional (A), debido a que el logaritmo neperiano de cero tiende a infinito, se empleó la Función Gamma Mixta (FGM) propuesta por Thom (1971) y Wu, Hayes, Wilhite y Svoboda (2005).

De lo antes expuesto surge la necesidad de caracterizar el fenómeno de la sequía meteorológica, obteniendo resultados que coadyuvarán a la identificación de las áreas vulnerables a la sequía en territorios agrícolas, donde se encuentran importantes superficies de cultivos de secano en el país; además, este estudio representa una aportación importante para el avance del conocimiento de la sequía en el ámbito agrícola. El objetivo de este estudio fue analizar la ocurrencia de la sequía meteorología en cuatro localidades de importancia agrícola del país por medio del SPI, haciendo uso de la combinación de métodos multivariados.

Debido a que las sequías configuran un gran número de patrones en el contexto espacial y temporal, resulta inviable caracterizarles de forma individual. Sin embargo, éstos pueden reducirse a unos pocos patrones, empleando dos técnicas estadísticas, en orden sucesivo: i) un Análisis de Coordenadas Principales y ii) un Análisis de Conglomerados. La combinación de estos métodos multivariados permitiría identificar los patrones espaciales mensuales dominantes que estructura el SPI sobre las localidades agrícolas estudiadas, y que, además, estarían relacionados con la ocurrencia de sequías locales de gran importancia.

\section{MÉTODOS}

La unidad de estudio estuvo constituida por las localidades de: El Tigre (Anzoátegui), Banco de San Pedro (Guárico), Tapipa Padrón (Miranda) y El Guayabo (Zulia). (Fig. 1). Los datos de precipitación mensual de la serie temporal de estudio fueron obtenidos de las estaciones 

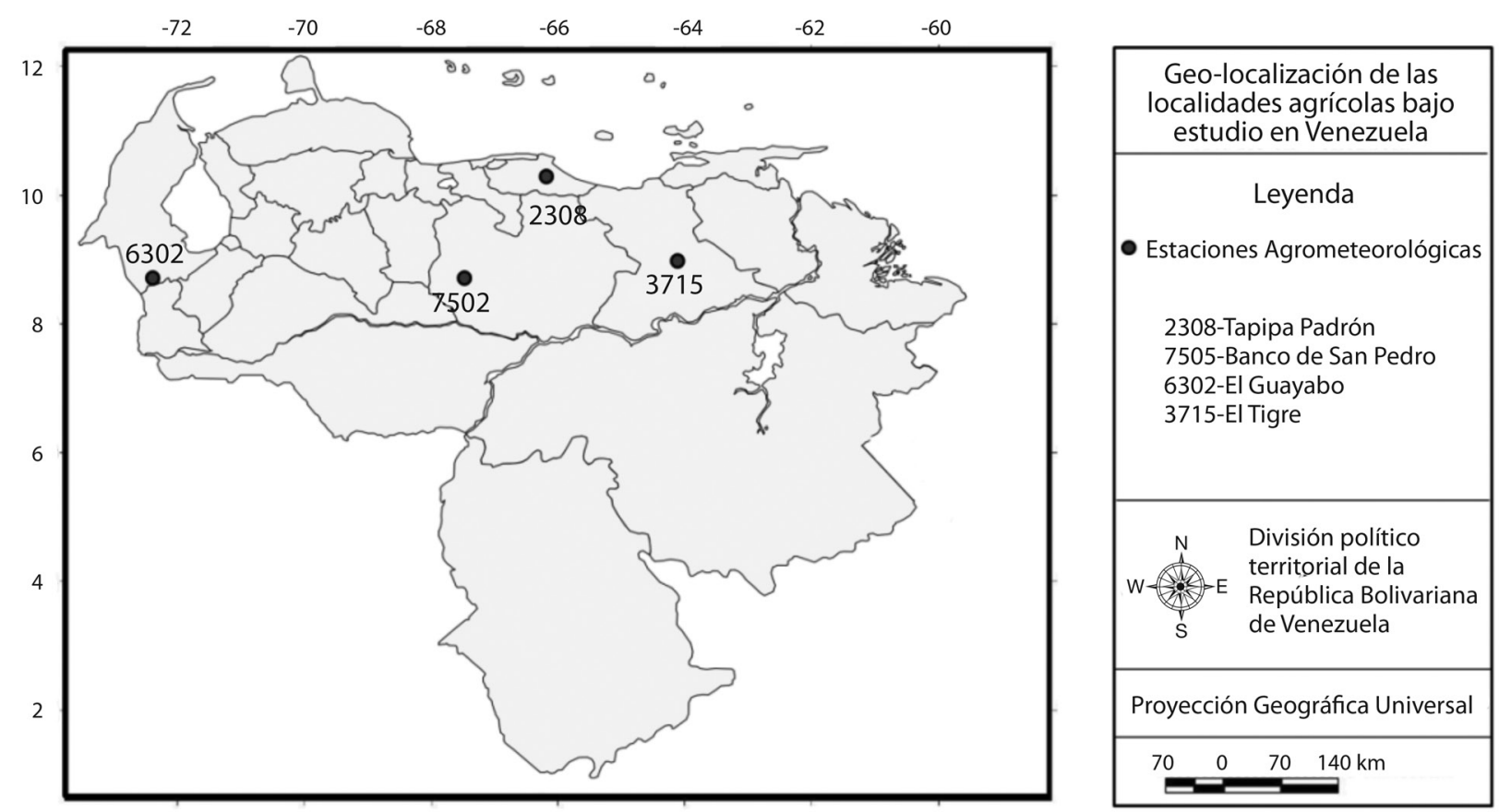

Fig. 1. Localización de las estaciones agrometeorológicas utilizadas en el estudio.

agrometeorológicas ubicadas en cada localidad agrícola analizada; dichas estaciones conforman la red de estaciones del Instituto Nacional de Investigaciones Agrícolas.

La selección de estas cuatro localidades estuvo en función al grado de vulnerabilidad agrícola a la sequía meteorológica, que va desde altamente vulnerables a medianamente vulnerables, según el estudio desarrollado por Olivares, Cortez, Parra, Lobo, Rodríguez y Rey (2017), el cual describe la evaluación de la vulnerabilidad agrícola a la sequía meteorológica para diferentes localidades agrícolas venezolanas.

Las localidades El Tigre y Banco de San Pedro se ubican en la zona subhúmeda de trópico bajo mientras que las localidades de Tapipa Padrón y El Guabayo pertenecen a la zona húmeda de trópico bajo (MARN, 2005).

En Tapipa Padrón predominan áreas de selva tropical poco intervenida y protegida para la conservación de la biodiversidad y de los recursos hidrológicos, con agricultura de subsistencia (conucos migratorios). Por otra parte, El Tigre se caracteriza por el desarrollo de la ganadería bovina extensiva y semi-intensiva; cultivos anuales mecanizados (cereales, leguminosas y oleaginosas); frutales comerciales (cítricos, mango y guayaba); Hortícolas de piso bajo; Agricultura de subsistencia y semi-comercial (Yuca, maíz, frijol, ajíes y otras hortalizas en pequeñas superficies).
En Banco de San Pedro predomina la agricultura de subsistencia y semi-comercial en vegas y algunas áreas altas; pesca y acuicultura; cereales con alta mecanización y uso de insumos. Por último, en El Guayabo predominan algunas áreas con explotaciones de cacao, plátanos y palma aceitera; ganadería bovina semi-intensiva y agricultura de subsistencia semi-comercial.

Se realizó un control de calidad a los datos de precipitación mensual para determinar la proporción de datos faltantes, identificar los valores atípicos y visualizar la distribución de las series (Parra \& Cortez, 2005; Ablan, Andressen, Vargas, \& Acevedo, 2008; Olivares, Cortez, Parra, Rodríguez \& Guevara, 2013); utilizando los programas estadísticos JMP v.6 (Jones \& Sall, 2011), InfoStat v.11 (Di Rienzo, Casanoves, Balzarini, González, Tablada \& Robledo, 2011) y Vesper v.1.6 (Minasny, McBratney \& Whelan, 2002).

Se utilizó el SPI, el cual cuantificó las condiciones de déficit o exceso de precipitación en las localidades agrícolas para una escala mensual del periodo 1980-2014, cuya metodología es reportada por Olivares et al. (2016a), Olivares, Cortez, Rodríguez, Parra, Lobo y Rey (2016b) y Olivares (2017). Los criterios de selección de este periodo de tiempo fueron la confiabilidad y calidad de las series de datos de precipitación mensual.

El índice representa el número de desviaciones estándar en que el valor transformado de la precipitación se 
desvía del promedio histórico, representado por el valor cero. Los valores negativos del SPI representan déficit de precipitación y aquellos valores positivos están asociados a datos de precipitación superiores al promedio histórico.

Para el análisis de los datos, en primer lugar, se ejecutó la imputación de datos faltantes utilizando un método basado en la Descomposición en Valores Singulares (Schmitt, Mandel \& Guedj, 2015). Posteriormente, se realizó el Análisis de Coordenadas Principales (o Escalamiento Multidimensional Métrico) de las matrices de datos usando distancia Euclídea (Kruskal \& Wish, 1978) y finalmente, se realizó un Análisis de Conglomerados sobre las dos primeras coordenadas principales usando el algoritmo de Ward (Kaufman \& Rousseeuw, 2009). Todos los análisis de datos se realizaron usando el paquete estadístico InfoStat (Di Rienzo et al., 2011).

El escalamiento métrico multidimensional, es un método que tiene como premisa la existencia de un isomorfismo entre las relaciones relativas de los objetos en el espacio original y las relaciones y medidas de los objetos en el espacio euclídeo; es decir, se asume que el método preserva las relaciones del espacio original en el nuevo espacio métrico y dos individuos próximos en el espacio $n$ - dimensional, se deben proyectar conjuntamente (Borg \& Groenen, 2005).

En consecuencia, las observaciones proyectadas en las dos primeras coordenadas principales son la mejor aproximación bidimensional de los patrones globales de sequía para cada conjunto de estaciones. Por otro lado, se determinaron conglomerados usando la información retenida por las dos primeras coordenadas, con el principal objetivo de caracterizar los patrones de sequía. El número de conglomerados fue determinado de acuerdo a la información proporcionada por el coeficiente de silueta (Rousseeuw, 1987).

De acuerdo a los resultados obtenidos en el último paso, en las cuatro localidades, se escogieron dos o tres grupos de años de SPI. En el caso de las localidades que resultaron con tres grupos, estos se categorizaron en años húmedos, intermedios o condiciones normales y años con déficit hídrico significativo. En tanto que, en el caso de las localidades que resultaron con dos grupos, se clasificaron en años húmedos y secos o asociados a déficit hídrico. Las categorías se escogieron de acuerdo a la media del índice dentro de cada grupo: en el caso de dos grupos, la media más elevada corresponde a años con

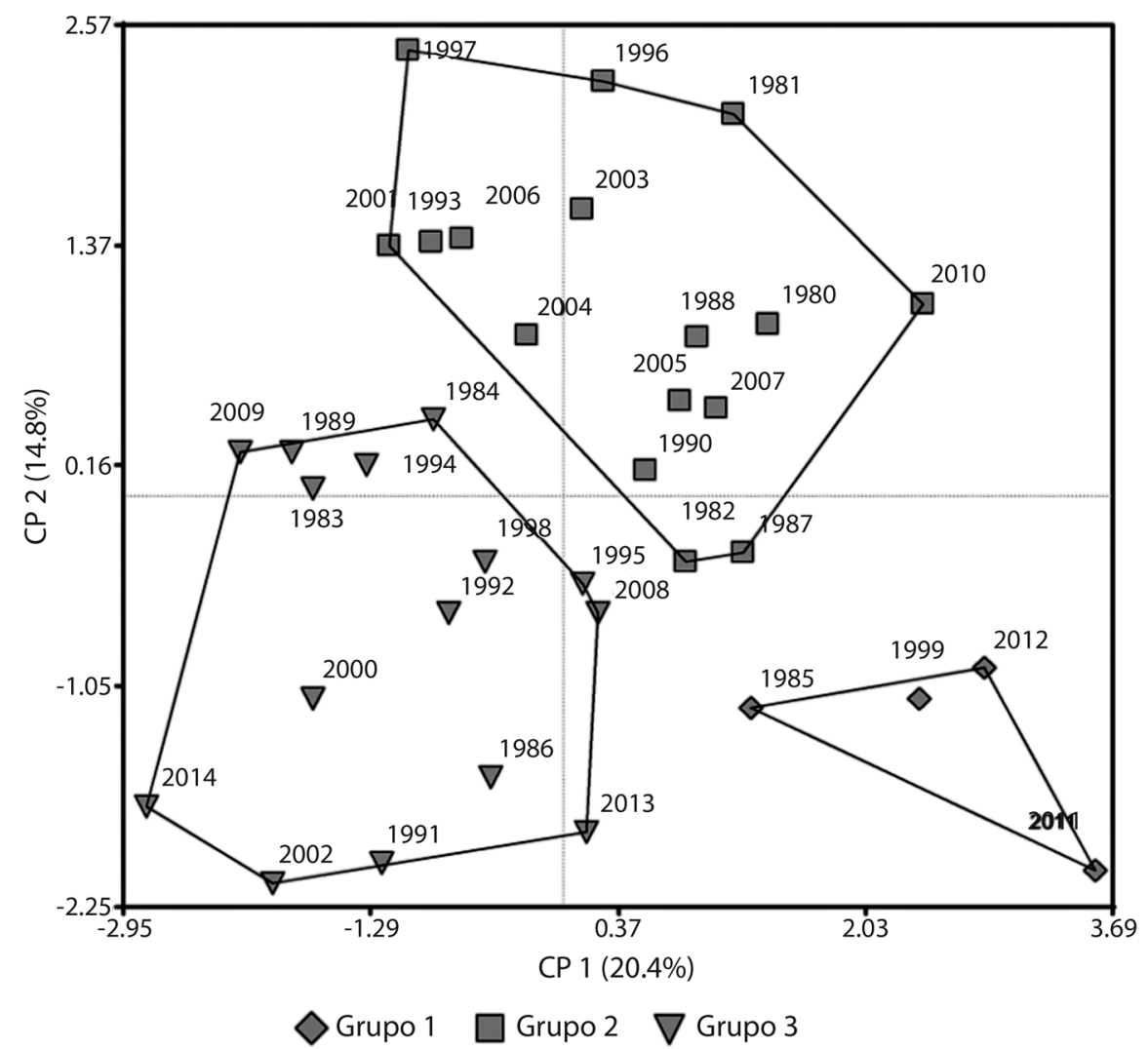

Fig. 2. Combinación del análisis de coordenadas principales y análisis de conglomerados para la serie temporal 1980-2014 del SPI en El Tigre estado Anzoátegui. 
más humedad; en tanto que, en el caso de tres grupos, el valor de la media más elevada corresponde a "húmedo", el valor intermedio a "condiciones normales" y el valor más pequeño a "años secos".

Para obtener una idea de la severidad de la sequía en los territorios agrícolas, se utilizó el Índice de Estrés Agrícola (ASI, siglas en inglés), el cual es un indicador desarrollado por la FAO que pone en relieve anomalías en el crecimiento de la vegetación y sequías potenciales en las áreas de cultivo durante una determinada temporada agrícola (Rojas, Vrieling \& Rembold, 2011).

\section{RESULTADOS}

Localidad El Tigre estado Anzoátegui: Para esta localidad Ilanera, se puede observar en la figura 2, que el primer eje (20.4\% de varianza explicada) es el que separa los tres grupos de años de la serie temporal. Los años más húmedos son los que tienen valores positivos en el eje x (grupo 1), los intermedios, son los representados en el grupo 2 (aproximadamente el 75\% de los años tienen los valores distribuidos alrededor del cero en el eje $\mathrm{x}$ ) y los más secos son los representados en el grupo 3, tienen los valores más pequeños (todos negativos, excepto los años 1995, 2008 y 2013) en el eje $x$.

El cuadro 1, presenta la categorización de los años basada en la similitud de las series mensuales del SPI para El Tigre, donde el año 2011 fue el más húmedo y el 2014, el más seco de toda la serie de SPI. El grupo 2 posee la mayor proporción de años ubicados en la categoría de años con condiciones normales, en función a los montos de lluvia recibidos en la zona.

Localidad Banco de San Pedro estado Guárico: Los años de la serie temporal en esta localidad, también se agruparon en tres grupos. Las dos primeras coordenadas, representan el 27.6 y $19.5 \%$ de la variabilidad total, lo que en la suma global es $47.1 \%$. En este caso, el primer eje, divide al grupo 3 de los otros dos. En tanto que, las diferencias de los grupos 1 y 2, sólo se ven en el segundo eje (Fig. 3).

El año 1981, se presenta a una mayor distancia del resto, por presentar un alto valor del índice (que podría considerarse atípico) en el mes de febrero, siendo superior al doble que el más alto para el mismo mes de los demás años. Adicionalmente, los años correspondientes al grupo 2 tienen una gran variabilidad, es decir, es un grupo muy heterogéneo y está conformado por años que han sido más húmedos y otros, más secos, de hecho, el solapamiento de los contornos, establece que no existen claras diferencias entre algunos de los años del grupo de 1 y 2.

El cuadro 2, presenta la categorización de los años basada en la similitud de las series mensuales del SPI para Banco de San Pedro, donde el grupo más homogéneo es el de los años más secos (grupo 3). También el grupo 2, posee la mayor proporción de años ubicados en la categoría de condiciones normales, en función del SPI.

Localidad Tapipa-Padrón estado Miranda: Las series de SPI derivadas de la estación Tapipa-Padrón, se dividen en dos grupos. El primer eje separa ambos grupos. Los valores negativos de este eje se corresponden con años más húmedos y los positivos, con años más secos (Fig. 4).

El cuadro 3, presenta la categorización de los años evaluados para esta localidad, siendo el grupo 1, el que posee mayor proporción de años en la serie temporal de SPI; esto asociado a los montos altos de lluvia recibidos históricamente en la zona, reflejados en el valor positivo que presentó el SPI.

Localidad El Guayabo estado Zulia: En la zona, la serie temporal se divide en dos grupos. El primer eje es el que separa estos grupos. Los valores negativos de este eje se corresponden con años más húmedos y los positivos con años más secos (Fig. 5).

\section{CUADRO 1}

Categorización de los años basada en la similitud de las series mensuales del SPI mediante la combinación de métodos multivariados en El Tigre.

\begin{tabular}{cll}
$\begin{array}{c}\text { Grupo } \\
\text { Grupo } 1\end{array}$ & \multicolumn{1}{c}{ Años } & \multicolumn{1}{c}{ Característica } \\
Grupo 2 & $\begin{array}{l}1985,1999,2011,2012 \\
1980,1981,1982,1987,1988,1990,1993,1996,\end{array}$ & $\begin{array}{l}\text { Se clasifican como años con altos valores del SPI. Años más } \\
\text { húmedos de la serie temporal. } \\
\text { Se clasifican como años con valores intermedios del SPI. Años } \\
\text { moderadamente secos. }\end{array}$ \\
Grupo 3 & $\begin{array}{l}1987,2001,2003,2004,2005,2006,2007,2010 \\
1998,2000,2002,2008,2009,2013,2014\end{array}$ & $\begin{array}{l}\text { Se clasifican como años con valores bajos del SPI. Años con } \\
\text { prevalencia de déficit hídrico en la serie temporal. }\end{array}$ \\
\hline
\end{tabular}




\section{CUADRO 2}

Categorización de los años basada en la similitud de las series mensuales del SPI mediante la combinación de métodos multivariados en Banco de San Pedro.

\begin{tabular}{cll} 
Grupo & \multicolumn{1}{c}{ Años } & \multicolumn{1}{c}{ Característica } \\
Grupo 1 & $1980,1984,1985,1988,1991,1992,2003,2004,2006$ & Años más húmedos de la serie temporal. \\
Grupo 2 & $1981,1982,1983,1987,1989,1993,2002,2005,2007,2008$ & Años moderadamente húmedos \\
Grupo 3 & $1986,1990,1999,2000,2001,2009$ & Años con prevalencia de déficit hídrico en la serie temporal \\
\hline
\end{tabular}

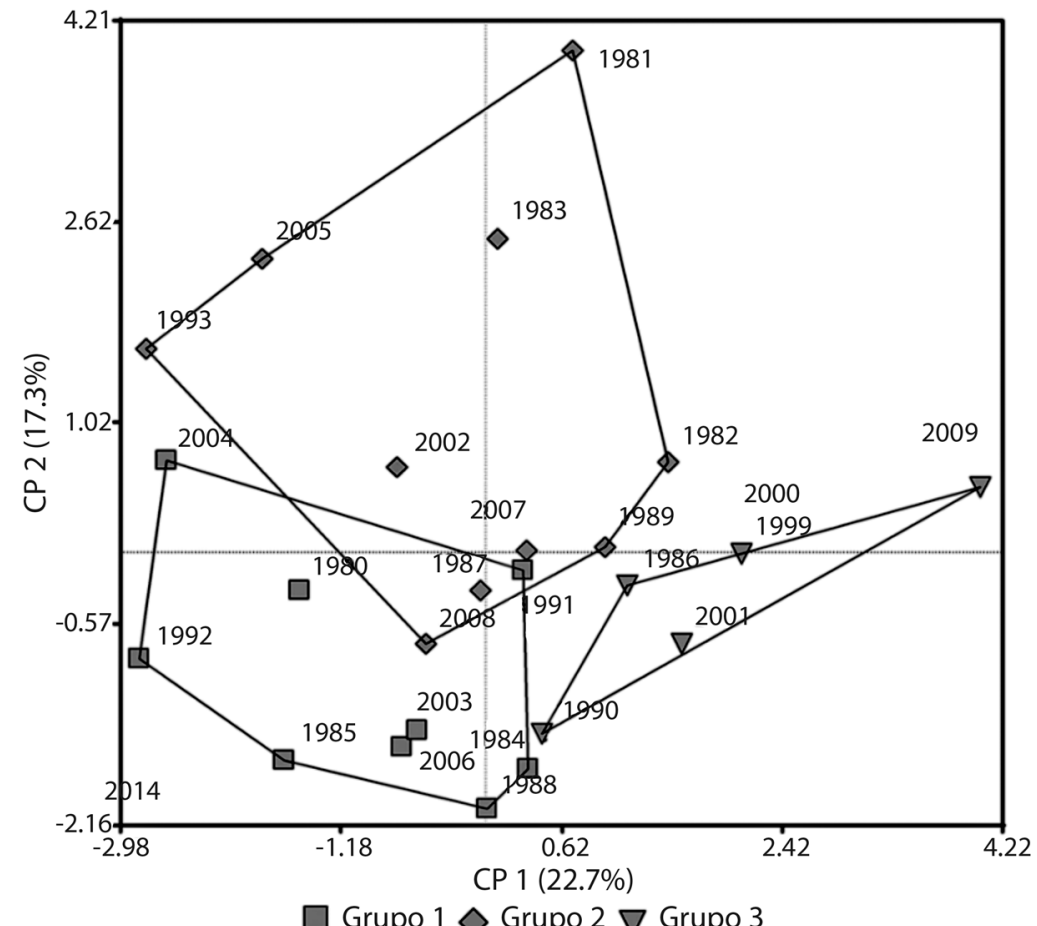

Fig. 3. Combinación del análisis de coordenadas principales y análisis de conglomerados para la serie temporal 1980-2014 del SPI en Banco de San Pedro estado Guárico.

\section{CUADRO 3}

Categorización de los años basada en la similitud de las series mensuales del SPI mediante la combinación de métodos multivariados en Tapipa-Padrón.

Grupo Años Característica

Grupo 1 1981, 1989, 1990, 1992, 1993, 2002, 2004, 2005, 2006, 2011, 2012, 2013 Años más húmedos de la serie temporal.

Grupo 2 1980, 1986, 1987, 1988, 1991, 2001, 2003, 2007, 2008, 2010, 2014

Años con prevalencia de déficit hídrico en la serie temporal 


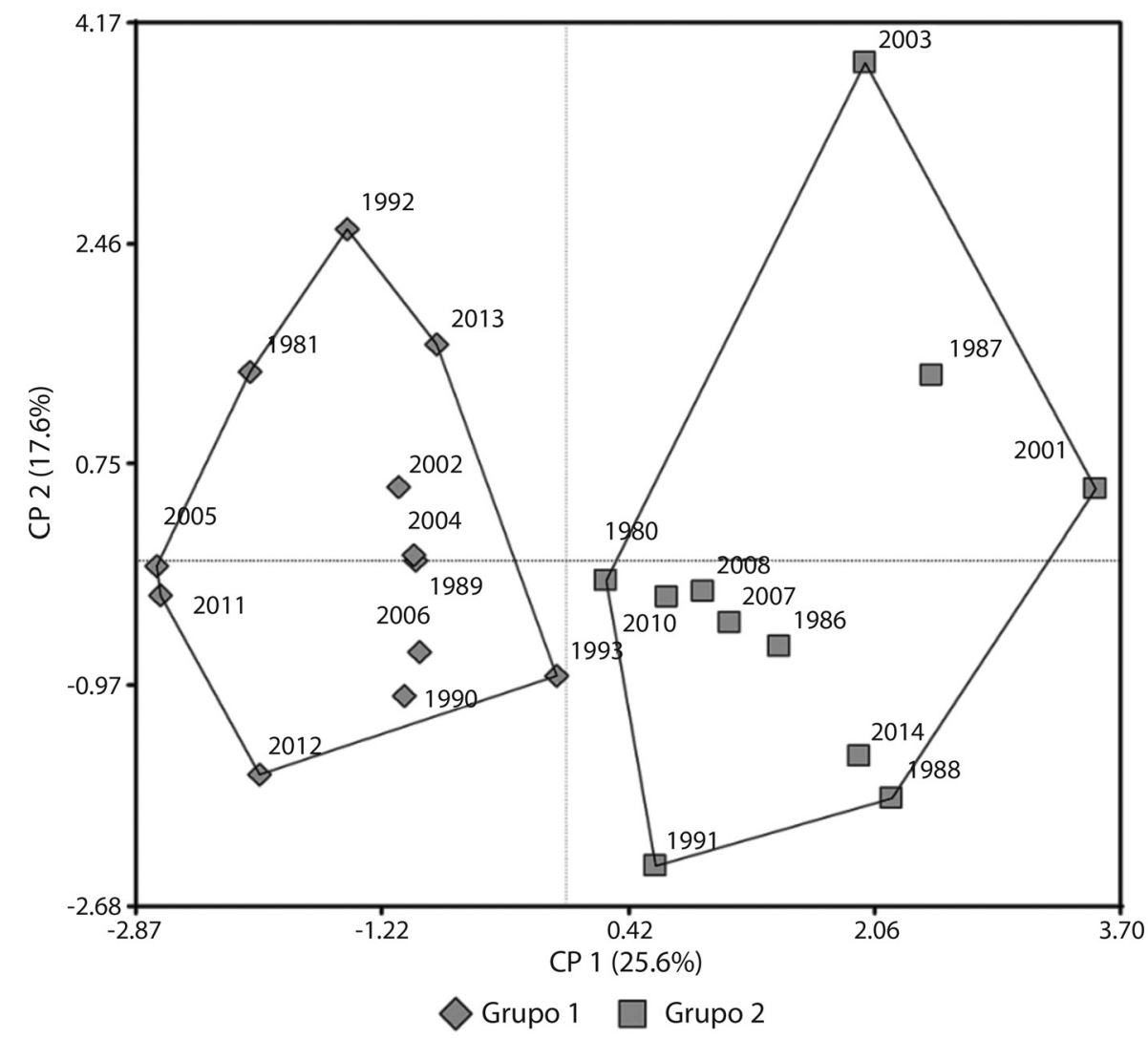

Fig. 4. Combinación del análisis de coordenadas principales y análisis de conglomerados para la serie temporal 1980-2014 en Tapipa-Padrón estado Miranda.

CUADRO 4

Categorización de los años basada en la similitud de las series mensuales del SPI mediante la combinación de métodos multivariados en El Guayabo.

\begin{tabular}{|c|c|c|}
\hline Grupo & Años & Característica \\
\hline Grupo 1 & $\begin{array}{l}\text { 1988, 1989, 1990, 1993, 1995, 1996, 1998, 1999, 2003, 2006, } \\
\text { 2007, 2008, 2009, 2010, } 2011\end{array}$ & Años más húmedos de la serie temporal. \\
\hline Grupo 2 & 1987, 1991, 1994, 2000, 2001, 2004, 2005, 2012, 2013, 2014 & $\begin{array}{l}\text { Años con prevalencia de déficit hídrico en la serie } \\
\text { temporal }\end{array}$ \\
\hline
\end{tabular}

El cuadro 4, presenta la categorización de los años para esta localidad, siendo el grupo 1 el que presenta mayor proporción de años de la serie temporal.

\section{DISCUSIÓN}

Para la localidad de El Tigre ubicada en los Llanos Orientales venezolanos, el período lluvioso es el más corto en comparación con las demás localidades bajo estudio, durando apenas seis meses (mayo a octubre), con promedio de lluvia anual de $1094 \mathrm{~mm}$. De acuerdo a los valores de SPI, la localidad EI Tigre pasó de 800-1200mm a 400-800mm en algunos años de la serie temporal. Esta disminución se evidenció durante el transcurso de la época lluviosa, generando consecuencias importantes tanto en el crecimiento, desarrollo y rendimiento de los cultivos como en la oportunidad de realizar las labores mecanizadas en campo.

La ocurrencia del déficit hídrico en la zona impactó la ya extraordinaria demanda de riego que tiene esta zona para los frutales mencionados y la producción de semilla, 


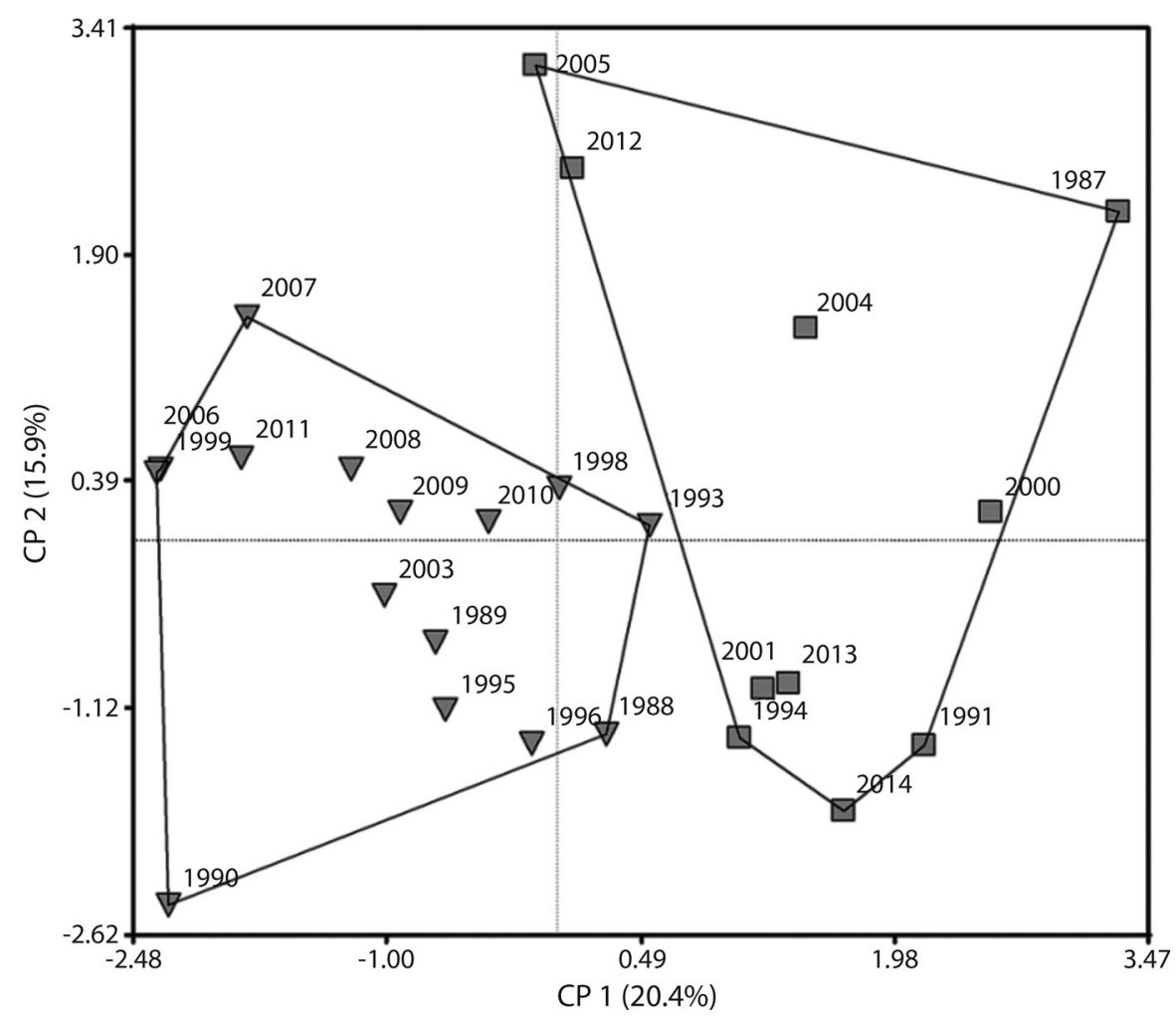

Grupo $1 \square$ Grupo 2

Fig. 5. Combinación del análisis de coordenadas principales y análisis de conglomerados para la serie temporal 1980-2014 en El Guayabo estado Zulia.

por lo arenosos que son estos suelos. Las escasas fuentes de agua subterránea exigirán nuevos esquemas de fuentes y métodos de riego (MARN, 2005; MPPAT, 2008).

Los resultados indican un alto nivel de afectación para El Tigre, cuyos usos agrícolas de yuca y pastos, estarían ubicados en áreas muy marginales por el déficit de humedad, lo que exigiría el riego complementario para mantenerlos en la zona.

En los Llanos Orientales venezolanos, la ocurrencia de estas sequías meteorológicas asociadas al cambio climático aumenta la fragilidad de la zona por la disminución de la precipitación; de esta manera, Ovalles et al. (2008), establecen que desaparecerán a largo plazo, prácticamente las zonas húmedas a expensas de zonas subhúmedas y gran parte de las zonas subhúmedas se convertirán en zonas secas con baja precipitación

Por su parte, la localidad Banco de San Pedro está ubicada en los Llanos Centrales (Guárico) cuyo período lluvioso es de seis a siete meses, de mayo a octubre, con montos de lluvia promedio anual de $1281 \mathrm{~mm}$. En los años más secos, la precipitación anual pasó de $1200 \mathrm{~mm}$ a $800 \mathrm{~mm}$, concentrándose dicha disminución durante la estación de crecimiento de los cultivos.

El estado Guárico, presenta una superficie cosechada de 50211 ha donde el $94 \%$ corresponde a los cereales, el $6 \%$ restante está distribuido entre cacao, plátano, caña, cambur, tabaco, pimentón, tomate y otras hortalizas (MPPAT, 2008). La principal zona afectada por el déficit hídrico fue el norte y al sur del estado, influyendo sobre la producción de hortalizas, frutales y semillas bajo riego del río Guárico, ello perjudicó principalmente la demanda de riego de una fuente ya crítica.

A raíz de esto, Hare y Ogallo (1993) así como Paredes, Barbosa, Ruiz y Peñaloza-Murillo (2016) reseñan que los procesos atmosféricos que conducen a la sequía son muy complejos, destacando la escasez de humedad atmosférica, la insuficiencia de sistemas generadores de lluvia, la persistencia de una fuerte subsidencia o una combinación de alguno de los factores mencionados.

Venezuela es uno de los países débilmente afectado por el fenómeno El Niño debido a que el sistema climático tiene una menor dependencia del Océano Pacífico. 
De acuerdo con estudios realizados por CONICIT (1998), Martelo (2000), Cárdenas et al. (2002), Martelo (2003), Tim y De Guenni (2016) se puede afirmar que los eventos El Niño en el país están relacionados con situaciones de sequía e incremento de la temperatura. Se podría establecer que sobre el territorio nacional se mezclan situaciones de influencia de la ZCIT del Atlántico y del Pacífico, combinaciones de vaguadas en altura, y restos de frentes fríos de origen Sur y Norte.

Para el periodo analizado, los resultados descritos evidencian que el fenómeno ENOA (El Niño-Oscilación Austral) puede explicar de forma parcial la ocurrencia de meses extremadamente secos o Extremos húmedos a escala local durante las temporadas húmedas y secas en distintas localidades de los llanos venezolanos (Paredes, Guevara-Pérez, Barbosa-Alves \& Uzcátegui-Briceño 2015; Olivares et al., 2016a).

Según Cárdenas et al. (2002), El Niño presenta una mayor incidencia sobre los extremos pluviométricos que La Niña a escala local y suele estar relacionado con condiciones anómalamente secas con moderada cobertura espacial. La moderada a escasa conexión entre las fases activas de ENOA identificada a escala local sugiere que otros factores no evaluados incidieron de forma determinante sobre ciertos eventos pluviométricos extremos.

Los resultados arrojados en esta investigación coinciden con los reportados por Hernández (2008), quien usó el SPI con agrupación semestral y mensual para caracterizar la sequía en los Llanos Centro Orientales de Venezuela.

Del mismo modo, La Cruz, Guevara y Paredes (2014), establecieron que las zonas del Sur de Anzoátegui y el Centro de Guárico son las regiones con mayor probabilidad de ocurrencia de años secos en la unidad de estudio constituida por localidades de importancia agrícola de los llanos venezolanos. Las zonas con mayor recurrencia de años secos también concuerdan con las reportadas en el estudio por Paredes, La Cruz y Guevara (2014).

En este orden de ideas, la localidad Tapipa-Padrón, estado Miranda, está ubicada en la zona húmeda de trópico bajo, presenta montos promedios de precipitación anual de $2623 \mathrm{~mm}$ distribuidos en más de ocho meses húmedos (abril a noviembre). Al disminuir el rango de lluvia por sequía de 1600-2000 a 800-1200mm en algunos años de la serie temporal, los cultivos importantes en la zona como cacao fueron afectados en rendimientos, aparición de plagas o enfermedades, y consecuentemente en su rentabilidad (INIA, 2016).

El estado Miranda, presenta una superficie cosechada de 32162 ha donde el $66 \%$ corresponde al cacao, el 16\% al cambur, el $14 \%$ al plátano, el $3 \%$ a los cereales y el restante $1 \%$ distribuido entre pimentón y tomate (MPPAT,
2008). Este último registra un rendimiento de $17145 \mathrm{~kg} /$ ha y el cacao $268 \mathrm{~kg} / \mathrm{ha}$, rendimientos afectados severamente en los años 1991, 2001, 2007 y 2014, cuyas reducciones estuvieron alrededor del $25 \%$.

Por otra parte, El Guayabo recibe un monto promedio de lluvia de $2083 \mathrm{~mm}$, con más de nueve meses húmedos (marzo-noviembre). En la región del Lago de Maracaibo, específicamente la zona de El Guayabo, se evidencia la disminución de la precipitación de $1200-1600 \mathrm{~mm}$ a 800-1 200mm en algunos años de la serie temporal. Los meses húmedos pasaron de cinco meses a tres o cuatro meses, debido al retraso en la entrada de lluvias.

Los rubros más afectados por la sequía meteorológica en la región del Sur del Lago de Maracaibo fueron la palma aceitera, plátanos, cacao y pastos. Los plátanos representan el $74 \%$ de la superficie cosechada en esta región, con un rendimiento de $10575 \mathrm{~kg} / \mathrm{ha}$; la palma africana se cosecha en un $25 \%$ de la región y su rendimiento es de $12920 \mathrm{~kg} / \mathrm{ha}$, y finalmente el cacao, único en el mundo como es el Porcelana, solo se cultiva en un $1 \%$ del área y tiene un rendimiento de $429 \mathrm{~kg} / \mathrm{ha}$ (MPPAT, 2008).

En El Guayabo, se afectaron significativamente los rendimientos de la palma aceitera, plátanos y cacao, lo cual exigirá a largo plazo la implementación de métodos más eficientes de conservación de la humedad del suelo (Ovalles et al., 2005; Cortez et al., 2011).

Severidad de la sequía meteorológica: De acuerdo con los datos reportados por Rojas, Li y Cumani (2015), el fenómeno El Niño 1991-92 duró 14 meses, siendo el segundo más largo de los últimos 30 años, clasificado de intensidad moderada. Sin embargo, las condiciones previas a El Niño 1991-92 se consideraban neutras con valores del Índice de El Niño Oceánico (INO) cercanos a 0,4.

Dentro de las zonas más afectadas por la sequía durante la primera campaña agrícola de 1991, estuvieron: Estados Unidos, Nicaragua, Honduras y el Caribe (Haití y República Dominicana), Colombia y Venezuela. Teniendo en cuenta la magnitud de los impactos negativos provocados por El Niño 1991-92 en las zonas agrícolas de todo el planeta, se clasificó, desde un punto de vista agrícola, como el episodio más severo de las últimas décadas. En el caso de Venezuela, el porcentaje de zonas agrícolas afectadas por la sequía ocurrida en 1991, estuvo alrededor del 40-50\%, siendo las localidades de El Tigre y Tapipa Padrón, las más afectadas (Fig. 6).

Al comparar estas estimaciones de la FAO con los resultados arrojados en esta investigación, se evidencia que existe correspondencia entre ambos, en las localidades agrícolas anteriormente mencionadas el rendimiento 

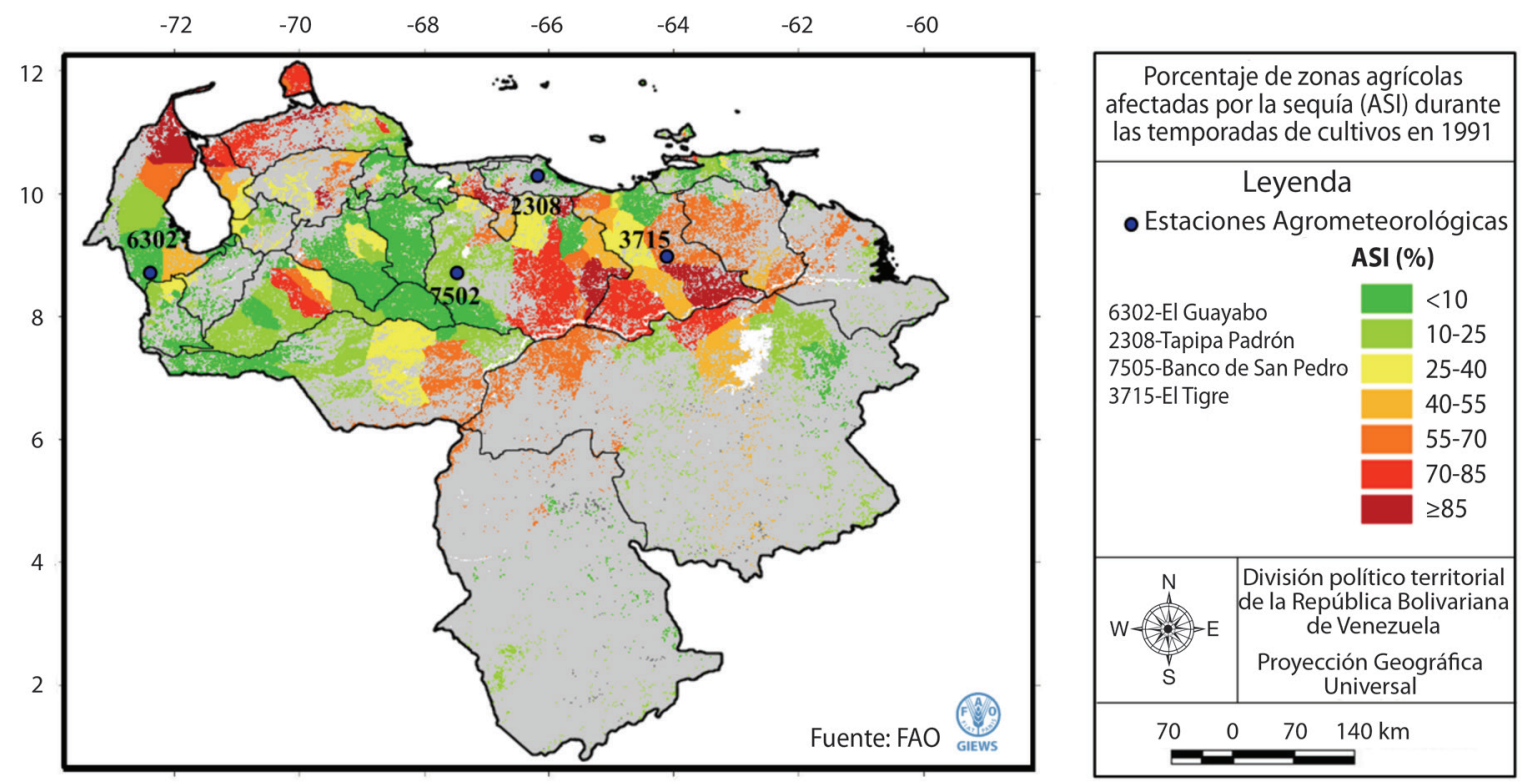

Fig. 6. Regiones afectadas por sequías según el índice ASI desarrollado por la FAO durante la primera campaña agrícola de 1991 en Venezuela. Fuente: Adaptado de FAO, 2015.

de los cereales disminuyo en un 40-50\% según cifras del MPPAT (2008).

La metodología estadística basada en el análisis multivariado en este estudio pudo delinear grupos de años que mostraron patrones semejantes en cuanto a la evolución del SPI mensual. El índice de sequía analizado fue lo suficientemente robusto, es decir, expresó la habilidad para analizar la sequía en un amplio rango de condiciones climáticas en Venezuela.

El impacto de la sequía meteorológica no es igual en todos los agroecosistemas, depende principalmente de las características biofísicas del sistema de producción representadas por la especie, cultivos y condiciones del suelo en la zona, de las capacidades tecnológicas y las condiciones socioeconómicas, entre otros.

El establecimiento de medidas de adaptación generales ante los eventos de sequía, aplicables a los distintos agroecosistemas aquí estudiados, será de acuerdo a las características propias de cada localidad agrícola.

El análisis de la sequía constituye la columna vertebral para la planificación del espacio y el fortalecimiento de las estrategias para la seguridad alimentaria.

Los datos aquí reportados sirven para que los organismos pertinentes consideren ciertas medidas preventivas y mitigantes en territorios agrícolas.

\section{AGRADECIMIENTOS}

La realización de esta investigación fue posible, gracias al apoyo de las investigadoras Adriana Cortez y Raquel Parra en la elaboración de la matriz de datos de lluvia y el cálculo del SPI. A ellas un agradecimiento sincero.

\section{REFERENCIAS}

Ablan, M., Andressen, R., Vargas, M. P., \& Acevedo, M. (2008). Propuesta metodológica para el control de calidad de datos de precipitación. Agronomía Tropical, 58 (1), 57-60.

Bonsal, B., Wheaton, E., Chipanshi, A., Lin, C., Sauchyn, D., \& Wen, L. (2011). Drought Research in Canada: A Review. Atmosphere-Ocean, 49(4), 303-319.

Borg, I., \& Groenen, P. J. (2005). Modern multidimensional scaling: Theory and applications. New York: Springer Science \& Business Media.

Cárdenas, P., García, L., \& Gil, A. (2002). Impacto de los eventos El Niño - Oscilación del Sur en Venezuela. Caracas: Corporación Andina de Fomento.

CONICIT (Consejo Venezolano de Investigaciones Científicas y Tecnológicas, Venezuela) (1998). El fenómeno El Niño y su posible influencia sobre el territorio de Venezuela. Caracas: CONICIT-CNMeH. 
Cortez, A., Rodríguez, M. F., Rey, J. C., Lobo, D., Parra, R., Ovalles, F., \& Gabriels, D. (2011). Análisis de la agresividad y concentración de las precipitaciones en Venezuela. II. Región noroccidental. Bioagro, 23 (1), 13-18.

Di Rienzo, J., Casanoves, F., Balzarini, M., González, L., Tablada, M., \& Robledo, C. (2011). InfoStat versión 2011. Grupo InfoStat, FCA, Universidad Nacional de Córdoba, Argentina. Recuperado de http://www.infostat.com.ar

Edwards, D. \& McKee, T (1997). Characteristics of 20th Century drought in the United States at multiple time scales. Atmospheric Science Paper No. 634. Climatology Report (97-2), USA: Colorado State University, Fort. Collins.

FAO (Organización de las Naciones Unidas para la Alimentación y la Agricultura, Italia). (2003). Manejo del riesgo en el uso del agua en la agricultura. En: Descubrir el Potencial del Agua para la Agricultura. Organización de las Naciones Unidas para la Alimentación y la Agricultura. Departamento de Desarrollo Sostenible. Roma, Italia. Recuperado de: http://www.fao.org/DOCREP/006/ Y4525S/y4525s07.htm.

FAO (Organización de las Naciones Unidas para la Alimentación y la Agricultura, Italia). (2015). Mapa de Índice de Estrés Agrícola (ASI) para Venezuela durante los años 1991, 2002, 2010, 2014 (en línea). Roma, Italia, FAO. s. esc. Color. Recuperado de http://www.fao.org/giews/ earthobservation

Guevara, E., \& Paredes, F. (2007). Influencia de variables macroclimáticas sobre el régimen pluviométrico en el estado Cojedes, Venezuela. Revista Ingeniería, UC, 14(3), 49-56.

Hare, F. K., \& Ogallo, J.A (1993). Climate variations, drought and desertification. WMO No. 653. Ginebra: WMO.

Hernández, R. (2008). Caracterización de la sequía meteorológica en los climas árido, semiárido y subhúmedo seco en los Llanos Centro Orientales de Venezuela, para el manejo de los recursos hídricos (en línea). Tesis MSc. Maracay, Venezuela, UNEFA. Recuperado de http:// www.inameh.gob.ve/documentos/Caracterizacion_sequia_Llanos_Centro_Orientales_Venezuela.pdf_

INIA (Instituto Nacional de Investigaciones Agrícolas, VE). 2016. Aproximación agroecológica para el nuevo modelo de producción agrícola en Venezuela. Maracay: Instituto Nacional de Investigaciones Agrícolas.

Jones, B., \& Sall, J. (2011). JMP statistical discovery software. Wiley Interdisciplinary Reviews: Computational Statistics, 3(3), 188-194.

Kruskal, J. B., \& Wish, M. (1978). Multidimensional scaling (Vol. 11). Sage. 96 p.

Kaufman, L., \& Rousseeuw, P. J. (2009). Finding groups in data: an introduction to cluster analysis (Vol. 344). Hoboken, New Jersey: John Wiley \& Sons.

La Cruz, F., Guevara, E., \& Paredes, F. (2014). Caracterización de las sequías meteorológicas en los llanos de Venezuela utilizando un análisis regional de frecuencias. Revista Ingeniería UC, 21(1), 70-76.
MARN (Ministerio del Ambiente y de los Recursos Naturales, Venezuela). (2005). Primera Comunicación Nacional en Cambio Climático de Venezuela. Caracas: PNUD-Fondo Mundial para el Medio Ambiente.

Martelo, M. T (2000). Estudio sobre la posible influencia del fenómeno El Niño - Oscilación del sur (ENOS) sobre el clima de los llanos de Venezuela. In OMM (eds.). Reunión de expertos de las asociaciones regionales AR-III y ARIV sobre fenómenos adversos (1, 2000, Ginebra, Suiza). Memoria. Ginebra, Suiza.

Martelo, M. T. (2003). Influencia de las variables macroclimáticas en el clima de Venezuela. Caracas: Ministerio del Ambiente y los Recursos Naturales, Dirección General de Cuencas Hidrográficas, Dirección de Hidrología, Meteorología y Oceanía.

Mckee, T., Doesken, N., \& Kleist, J. (1993). The relationship of drought frequency and duration to time scales. In American Meteorological Society (eds.). Conference on Applied Climatology, (8, 1993, Anaheim, California, USA). Proceedings. California, USA. AMS.

Minasny, B., McBratney, A., \& Whelan, B. (2002). Vesper version 1.6. Australian Centre for Precision Agriculture. The University of Sidney. Recuperado de http://www.usyd. edu.au/su/agric/acpa

MPPAT (Ministerio del Poder Popular para la Agricultura y Tierras, Venezuela) (2008). VII Censo Agrícola Nacional de Venezuela. Recuperado de http://censo.mat.gob.ve/

Olivares, B. (2017). La sequía meteorológica en territorios agrícolas de Venezuela: un análisis temporal del fenómeno meteorológico y su impacto en la agricultura venezolana. Saarbrücken, Alemania: Editorial Académica Española.

Olivares, B., Cortez, A., Lobo, D., Parra, R., Rey, J. C., \& Rodríguez, M. F. (2016a). Estudio de la Sequía Meteorológica en Localidades de los Llanos de Venezuela Mediante el Índice de Precipitación Estandarizado. Revista Acta Nova, 7(3), 266-283.

Olivares, B., Cortez, A., Parra, R., Lobo, D., Rodríguez, M. \& Rey, J.C. (2017). Evaluation of agricultural vulnerability to drought weather in different locations of Venezuela. Revista de la Facultad de Agronomía (LUZ), 34 (1), 103-129.

Olivares, B., Cortez, A., Parra, R., Rodríguez, M.F. \& Guevara, E. (2013). Aplicación de procedimientos estadísticos para el control de calidad de las series de precipitación mensual de los llanos orientales venezolanos. Revista de la Facultad de Agronomía, 30 (3), 367-391.

Olivares, B., Cortez, A., Rodríguez, M. F., Parra, R., Lobo, D., \& Rey, J.C. (2016b). Análisis temporal de la sequía meteorológica en localidades semiáridas de Venezuela. UGCiencia, 22(1), 11-24.

Ovalles, F., Cabrera-Bisbal, E., Cortez, A., Rodríguez, M., Rey, J. C., \& Comerma, J. (2005). Aproximación a los escenarios de adaptación al cambio climático del sector agrícola. Maracay: INIA. 
Ovalles, F., Cortez, A., Rodríguez, M. F., Rey, J. C., \& CabreraBisbal, E. (2008). Variación geográfica del impacto del cambio climático en el sector agrícola en Venezuela. Agronomía Tropical, 58 (1), 37-40.

Paredes, F., \& Guevara, E. (2010). Desarrollo y evaluación de un modelo para predecir sequías meteorológicas en Los Llanos de Venezuela. Revista Bioagro, 22(1), 3-10.

Paredes, F., Barbosa, H. A., Ruiz, I. Q., \& Peñaloza-Murillo, M. A. (2016). Meso scale oceanic atmospheric circulation patterns linked with severe and extensive droughts in Venezuela. Revista Brasileira de Meteorología, 31(4), 468-489. Recuperado de https://dx.doi. org/10.1590/0102-778631231420150070

Paredes, F., Guevara-Pérez, E., Barbosa-Alves, H., \& UzcáteguiBriceño, C. (2015). Tendencia de la precipitación estacional e influencia de El Niño-Oscilación Austral sobre la ocurrencia de extremos pluviométricos en la cuenca del lago. Tecnología y Ciencias del Agua, VI (6), 33-48.

Paredes, F., La Cruz, F., \& Guevara, E. (2014). Análisis regional de frecuencia de las sequías meteorológicas en la principal región cerealera de Venezuela. Revista Bioagro, 26 (1), 21-28.

Paredes, F., Millano, J. L., \& Guevara, E. (2008). Análisis espacial de las sequías meteorológicas en la región de Los Llanos de Venezuela durante el período 1961-1996. Revista Climatología de España, 8, 15-27.

Parra, R., \& Cortez, A. (2005). Control de calidad de series de precipitación de las series de precipitación del INIA Venezuela en el periodo 1970-2000. Rev. Arg. de Agrometeorología, 5-6, 63-73.

Quiroz, R. I., Paredes, F., \& Guevara, E. (2016). Incidencia de las sequías sobre las cuencas aportantes a los grandes embalses en Venezuela. Ágora de heterodoxias, 2, (3), 65-89.

Rojas, M., \& Alfaro, E. (2000). Influencia del océano Atlántico tropical sobre el comportamiento de la primera parte de la estación lluviosa en Venezuela. Tópicos Meteorológicos y Oceanográficos, 7(2), 88-92. Recuperado de http:// cglobal.imn.ac.cr/sites/default/files/documentos/revista/topicosmet20002/index.html

Rojas, O., Li, Y., \& Cumani, R. (2015). Entendiendo el impacto de sequía provocada por El Niño en el área agrícola mundial: una evaluación utilizando el Índice de Estrés Agrícola de la FAO (ASI). Roma: FAO.

Rojas O., Vrieling, A., \& Rembold, F. (2011). Evaluación de la probabilidad sequía en zonas agrícolas de África con imágenes de teledetección remota de basta resolución. Remote Sensing of Environment, 115, 343-352.

Rousseeuw, P. J. (1987). Silhouettes: a graphical aid to the interpretation and validation of cluster analysis. Journal of computational and applied mathematics, 20, 53-65.

Schmitt, P., Mandel, J., \& Guedj, M. (2015). A comparison of six methods for missing data imputation. Journal of Biometrics \& Biostatistics, 6(1), 224. doi: $10.4172 / 2155-6180.1000224$

Thom, H. (1971). Some methods of climatological analysis. Nota técnica No 81, OMM No 199, TP 103. Ginebra, Suiza: Secretaría de la OMM.

Tim, N., \& De Guenni, L. B. (2016). Oceanic influence on the precipitation in Venezuela under current and future climate. Climate Dynamics, 47(1-2), 211-234.

Wilhite, D., \& Glantz, M. (1985). Understanding the drought phenomenon: the role of definitions. Water International, 10, 111-120.

Wilhite, D. (1997). Improving drought management in the West. The Role of mitigation and preparedness. USA: National Drought Mitigation Center, Utah State University.

Wilhite, D. (2000). Drought as a natural hazard. En: Wilhite, D.A. (Eds.), Drought: A Global Assessment. London: Routledge Publishers.

Wu, H., Hayes, M. L., Wilhite, D. A., \& Svoboda, M. D. (2005). The effect of the length of record on the Standardized Precipitation Index calculation, Int. J. Climato, 25, 505-520. 